\title{
Long-Term Ivacaftor in People Aged 6 Years and Older with Cystic Fibrosis with Ivacaftor-Responsive Mutations
}

\author{
Joseph M. Pilewski $\cdot$ Kris De Boeck · Jerry A. Nick $\cdot$ Simon Tian • \\ Cynthia DeSouza $\cdot$ Mark Higgins $\cdot$ Richard B. Moss
}

Received: July 28, 2020 / Published online: September 23, 2020

(C) The Author(s) 2020

\section{ABSTRACT}

Introduction: Mutations in the cystic fibrosis transmembrane conductance regulator gene (CFTR) affect the quantity and/or function of

Digital Features To view digital features for this article go to https://doi.org/10.6084/m9.figshare.12841538.

Electronic supplementary material The online version of this article (https://doi.org/10.1007/s41030020-00129-2) contains supplementary material, which is available to authorized users.

J. M. Pilewski $(\bowtie)$

University of Pittsburgh, UPMC Montefiore

Hospital, NW628, 3459 Fifth Ave, Pittsburgh, PA

15213, USA

e-mail: pilewskijm@upmc.edu

K. De Boeck

Faculty of Medicine, University Hospital of Leuven, Herestraat 49, 3000 Leuven, Belgium

J. A. Nick

National Jewish Health, 1400 Jackson St, Denver, CO 80206, USA

S. Tian - C. DeSouza

Vertex Pharmaceuticals Incorporated, 50 Northern

Ave, Boston, MA 02210, USA

M. Higgins

Vertex Pharmaceuticals (Europe) Limited, 2

Kingdom St, London W2 6BD, UK

R. B. Moss

Center of Excellence in Pulmonary Biology,

Stanford University School of Medicine, 770 Welch

Rd \#350, Palo Alto, CA 94304, USA
CFTR protein reaching the cell surface. Ivacaftor, a CFTR potentiator that enhances chloride transport, increases the channel-open probability of normal and dysfunctional CFTR. Initially approved for people with CF (pwCF) with G551D-CFTR gating mutations, ivacaftor demonstrated clinical benefit in pwCF with other gating mutations and certain residual function mutations, including $R 117 H-C F T R$, in clinical studies. We evaluated the long-term safety and efficacy of ivacaftor in pwCF aged 6 years and older with non-G551D-CFTR ivacaftor-responsive mutations.

Methods: Efficacy and safety data from a phase 3 , multicenter, open-label, extension study for participants from Study 110 (R117H-CFTR mutations), Study 111 (non-G551D-CFTR gating mutations), and Study 113 (n-of- 1 pilot study in participants with residual CFTR function) were analyzed. Following washout from the randomized parent study, participants received oral ivacaftor $150 \mathrm{mg}$ once every $12 \mathrm{~h}$ for 104 weeks.

Results: Forty-one of 121 participants completed treatment through 104 weeks; 59 participants who did not complete the extension study continued treatment with commercial ivacaftor. The most common adverse events were pulmonary exacerbation (46.3\%) and cough (33.9\%). Most treatment-emergent adverse events were mild/moderate in severity and consistent with manifestations of CF or the ivacaftor safety profile. Rapid, durable 
improvement occurred across all efficacy endpoints.

Conclusions: Ivacaftor was generally safe and well tolerated with no new safety concerns for up to 104 weeks in pwCF with ivacaftor-responsive mutations. The pattern of improvement across efficacy endpoints was durable and generally consistent with parent-study outcomes.

Trial Registration: NCT01707290

Keywords: Ivacaftor; Long-term efficacy; Longterm safety; Non-G551D mutation; Residual function; R117H; Gating mutation

\section{Key Summary Points}

Why carry out this study?

Initially approved for people with G551D-

CFTR gating mutations, ivacaftor demonstrated clinical benefit in pwCF with other gating mutations and certain residual function mutations, including R117H-CFTR, in clinical studies.

We evaluated the long-term safety and efficacy of ivacaftor in pwCF aged $\geq 6$ years with non-G551D-CFTR ivacaftorresponsive mutations in a rollover extension of three parent studies.

What was learned from the study?

Ivacaftor was generally safe and well tolerated with no new safety concerns for up to 104 weeks in pwCF with non-G551DCFTR ivacaftor-responsive mutations.

The pattern of improvement across efficacy endpoints was durable and generally consistent with parent study outcomes.

\section{INTRODUCTION}

Cystic fibrosis (CF) is an autosomal recessive disease caused by mutations in the CF transmembrane conductance regulator gene (CFTR) that affect the quantity and/or function of CFTR protein at the cell surface [1].

Ivacaftor $\left(\right.$ Kalydeco $^{\circledR}$ ) is a CFTR potentiator that enhances chloride transport by increasing the channel-open (i.e., gating) probability of both wild-type and dysfunctional CFTR protein at the cell surface [2]. Ivacaftor was initially approved in 2012 for the treatment of people with CF (pwCF) with a G551D-CFTR gating mutation $[1,2]$. Since then, ivacaftor has demonstrated clinical benefit in more trials and in real-world data, and it is now approved in the United States for pwCF aged 6 months and older who have a mutation responsive to ivacaftor, including gating mutations and certain mutations associated with residual CFTR function (Appendix S1 in the supplement) [2-11]; currently approved genotypes and ages vary by country and region $[2,12]$. Ivacaftor-responsive mutations have demonstrated responsiveness to ivacaftor in vitro and/or in clinical data $[1,2,13]$.

This rollover extension study (Extension Study 112) was open to qualifying pwCF who had participated in one of three previous studies investigating the treatment effects of ivacaftor in CF: Study 110 (NCT01614457), in which participants had an R117H-CFTR mutation [8]; Study 111 (NCT01614470), in which participants had a non-G551D-CFTR gating mutation [6]; and Study 113, in which participants had phenotypic or molecular evidence of residual CFTR function (NCT01685801) [14].

The total duration of ivacaftor treatment in the three parent studies ranged from 12 to 24 weeks $[6,8,14]$. The primary objective of the present extension study was to evaluate the long-term safety of ivacaftor therapy in pwCF aged 6 years and older with ivacaftor-responsive mutations. The secondary objective was evaluation of long-term efficacy.

\section{METHODS}

\section{Participants and Study Design}

This was a phase 3, multicenter, open-label, extension study (NCT01707290) in pwCF who had previously enrolled in Study 110, Study 
111, or Study 113 (Fig. 1). Participants received oral ivacaftor $150 \mathrm{mg}$ once every $12 \mathrm{~h}$ for up to 104 weeks.

Participants entering from Study 110 or Study 111 must have completed their assigned study drug treatments in their respective parent studies. Participants entering from Study 113 must have completed study drug treatment through follow-up and met $\geq 1$ of 4 responder criteria. For full responder criteria, see Appendix S2 in the supplement. Exclusion criteria included a history of any illness or condition that, in the opinion of the investigator, might confound the results of the study or pose an additional risk in administering the study drug to the participant. For full inclusion and exclusion criteria, see Appendix S2 in the supplement.

This study was conducted in accordance with good clinical practices, as described in the International Council for Harmonisation guidelines, and is consistent with the World Medical Association Declaration of Helsinki and applicable regional laws and regulations. The study protocol, informed consent form, and other necessary documents were reviewed and approved by an independent ethics committee or institutional review board for each study site before initiation of the study at that site
(Table S1 in the supplement). Written informed consent was obtained from or for (with assent, where applicable) each participant on (or before) the day 1 visit.

\section{Outcomes}

The primary objective was to evaluate the longterm safety of ivacaftor treatment in pwCF aged 6 years and older with ivacaftor-responsive mutations. Safety outcomes included the incidence of treatment-emergent adverse events (AEs) and clinical laboratory measures. The secondary objective was to evaluate long-term efficacy. Secondary efficacy measures included mean absolute change from baseline in percent predicted forced expiratory volume in $1 \mathrm{~s}$ $\left(\mathrm{ppFEV}_{1}\right)$, sweat chloride concentration, respiratory domain score of the Cystic Fibrosis Questionnaire-Revised (CFQ-R RD), measures of nutritional status, and time to first pulmonary exacerbation (PEx).

\section{Statistical Methods}

The full analysis set (FAS) was defined as all participants who received $\geq 1$ dose of study drug during the extension study. All analyses

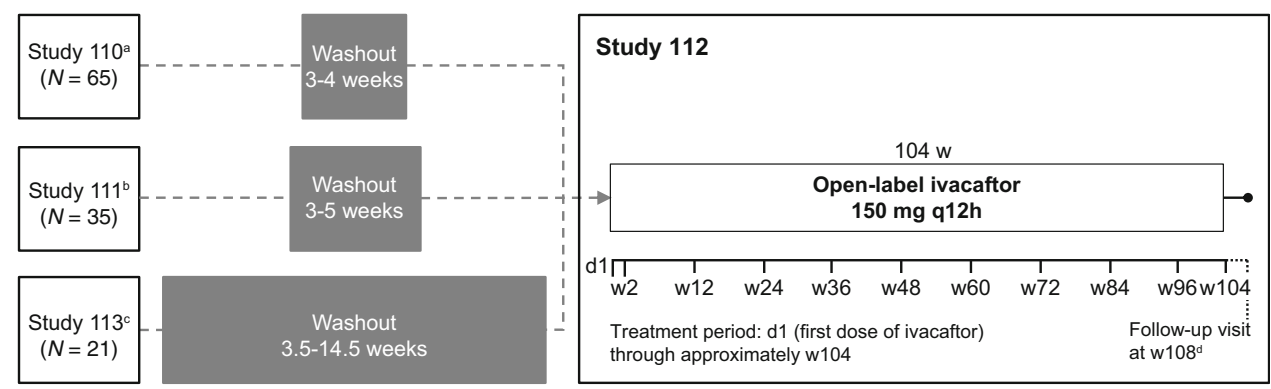

Fig. 1 Study design. a'Study 110 was a randomized controlled trial of ivacaftor vs. placebo for 24 weeks in pwCF who had an $R 117 H$-CFTR mutation; $\mathrm{N}$ is for the full analysis set. ${ }^{\mathrm{b}}$ Study 111 was a randomized crossover trial of ivacaftor vs. placebo for 8 weeks per treatment, followed by 16 weeks of open-label ivacaftor, in pwCF who had a non-G551D-CFTR gating mutation; $\mathrm{N}$ is for the full analysis set. ${ }^{\mathrm{C}}$ Study 113 was a randomized crossover trial of ivacaftor vs. placebo for a total of 4 weeks per treatment, followed by 8 weeks of open-label ivacaftor, in
pwCF who had phenotypic or molecular evidence of residual CFTR function; $\mathrm{N}$ is for the full analysis set. ${ }^{\mathrm{d}}$ Follow-up visit occurred 4 weeks ( \pm 7 days) after the last ivacaftor dose for participants who did not continue immediately on ivacaftor. If applicable, an early termination visit occurred as soon as possible after the last ivacaftor dose. CFTR cystic fibrosis transmembrane conductance regulator, $d$ day, $p w C F$ people with cystic fibrosis, $q 12 h$ once every $12 \mathrm{~h}, w$ week 
were performed for the FAS and are presented by parent-study subgroup. No power or sample size analyses were conducted. Descriptive analyses of safety were performed.

For the secondary efficacy outcomes, mixedeffects models for repeated measures and leastsquares (LS) means were used to assess absolute change from baseline in $\mathrm{ppFEV}_{1}$, sweat chloride concentration, and CFQ-R RD score. For body mass index (BMI), a linear mixed-effects model was used to assess the change from baseline over 104 weeks, and the LS mean of the rate of change over 104 weeks was used. For analyses presenting change from baseline, the Extension Study 112 baseline following washout from the parent study, which was defined as the most recent nonmissing (scheduled or unscheduled) measurement collected before initial administration of the study drug in Extension Study 112 , was used rather than that from the treatment period of the parent study. Kaplan-Meier methods were used to assess the time to first PEx by study day.

\section{RESULTS}

Of 121 participants who were enrolled and received ivacaftor in the extension study (Table 1; FAS shown in Figure S1 in the supplement), 41 (33.9\%) completed the full study drug treatment duration (104 weeks), and 35 (28.9\%) attended the follow-up visit 4 weeks ( \pm 7 days) after stopping ivacaftor. Of the 80 participants who did not complete the study drug treatment, most (59 [73.8\%]) discontinued study participation because they chose to receive commercially available ivacaftor, which had become available.

Most participants who rolled over from Study 110 had an F508del-R117H-CFTR genotype. Participants who rolled over from Study 111 and Study 113 had a variety of gating and residual function mutations, respectively, with no single dominant genotype; more than half of participants rolling over from Study 111 and Study 113 carried F508del on the second allele. A complete list of CFTR mutations in the FAS is shown in Table S2 in the supplement.

\section{Safety Outcomes (Primary Endpoint)}

Ivacaftor was generally safe and well tolerated in this study. Most participants experienced $\geq 1$ AE (Table 2). The most common treatmentemergent AEs were infective PEx of CF (46.3\%), cough $(33.9 \%)$, headache $(19.0 \%)$, sinus congestion and sputum increased (18.2\% each), nasopharyngitis $(17.4 \%)$, and sinusitis (15.7\%). Among the 27 participants who experienced serious AEs (SAEs), two had SAEs that were considered related to the study drug (Table 2). SAEs occurring in $>1$ participant were infective PEx of CF $(n=21[17.4 \%])$, gastroenteritis $(n=2$ $[1.7 \%])$, and pneumonia $(n=2[1.7 \%])$. Three participants (2.5\%) discontinued due to AEs. No deaths occurred. No clinically relevant trends in clinical laboratory test results attributable to ivacaftor treatment were apparent. Elevated transaminase levels were infrequent (Table S3 in the supplement).

\section{Secondary Efficacy Results}

Given the differences in CFTR genotypes across the parent studies, data are presented by parentstudy subgroups to provide a better understanding of efficacy by mutation type.

Improvements in $\mathrm{ppFEV}_{1}$ (LS mean absolute changes from baseline) were generally stable and durable over time during the 104 weeks of treatment, with an increase in variability in the magnitude of response during the latter part of the treatment period associated with the decreased number of participants (Fig. 2). However, the overall pattern of effects was consistent with results from the parent studies (Table S4 in the supplement).

In the 30 participants who completed the follow-up visit with a $\operatorname{ppFEV}_{1}$ measure, the gains in $\mathrm{ppFEV}_{1}$ during the treatment period trended back to pretreatment baseline levels in the 4-week follow-up period when they were no longer receiving ivacaftor.

LS mean changes from baseline in sweat chloride concentration varied considerably across parent-study subgroups (Fig. 3), with a robust response observed in participants from Study 111, a more modest response in 
Table 1 Baseline participant demographics and characteristics by parent-study subgroup

\begin{tabular}{|c|c|c|c|c|}
\hline Characteristic & $\begin{array}{l}\text { Study } 110 \\
R 117 H^{a} \\
(n=65)\end{array}$ & $\begin{array}{l}\text { Study } 111 \\
\text { Gating } \\
(n=35)\end{array}$ & $\begin{array}{l}\text { Study } 113 \\
\mathbf{R F}^{\mathrm{c}} \\
(n=21)\end{array}$ & All participants $(N=121)$ \\
\hline Male, $n(\%)$ & $28(43.1)$ & $22(62.9)$ & $10(47.6)$ & $60(49.6)$ \\
\hline White, $n(\%)$ & $65(100.0)$ & $32(91.4)$ & $21(100.0)$ & $118(97.5)$ \\
\hline \multicolumn{5}{|l|}{ Age } \\
\hline Mean (SD), years & $32.4(16.9)$ & $23.9(13.6)$ & $40.0(13.5)$ & $31.3(16.3)$ \\
\hline 6 to 11 years, $n(\%)$ & $13(20.0)$ & $8(22.9)$ & 0 & $21(17.4)$ \\
\hline 12 to 17 years, $n(\%)$ & $2(3.1)$ & $8(22.9)$ & $1(4.8)$ & $11(9.1)$ \\
\hline$\geq 18$ years, $n(\%)$ & $50(76.9)$ & $19(54.3)$ & $20(95.2)$ & $89(73.6)$ \\
\hline \multicolumn{5}{|l|}{ ppFEV $_{1}$} \\
\hline Mean (SD), percentage points & $71.8(20.4)$ & $78.3(21.1)$ & $62.8(22.2)$ & $72.1(21.4)$ \\
\hline$<70 \%, n(\%)$ & $29(44.6)$ & $12(34.3)$ & $15(71.4)$ & $56(46.3)$ \\
\hline$\geq 70 \%$ to $\leq 90 \%, n(\%)$ & $23(35.4)$ & $10(28.6)$ & $3(14.3)$ & $36(29.8)$ \\
\hline$>90 \%, n(\%)$ & $13(20.0)$ & $13(37.1)$ & $3(14.3)$ & $29(24.0)$ \\
\hline BMI, mean $(\mathrm{SD}), \mathrm{kg} / \mathrm{m}^{2}$ & $23.8(5.6)$ & $23.2(5.3)$ & $24.8(5.8)$ & $23.8(5.5)$ \\
\hline Sweat chloride concentration, mean (SD), mmol/l & $60.9(19.4)^{\mathrm{d}}$ & $80.2(22.8)^{\mathrm{e}}$ & $55.7(22.2)$ & $65.6(22.9)^{\mathrm{f}}$ \\
\hline
\end{tabular}

$B M I$ body mass index, $p p F E V_{1}$ percent predicted forced expiratory volume in $1 \mathrm{~s}, R F$ residual function

${ }^{a}$ Participants had an $R 117 H-C F T R$ mutation

b Participants had a non-G551D-CFTR gating mutation

${ }^{c}$ Participants had phenotypic or molecular evidence of residual CFTR function

d $N=59$

e $N=33$

${ }^{\mathrm{f}} \mathrm{N}=113$

participants from Study 110, and little to no response in participants from Study 113. There was a rapid improvement from baseline in CFQ$\mathrm{R}$ RD score across all parent-study subgroups (Figure S2 in the supplement). The improvements in participant-reported respiratory symptoms were stable and durable over the 104 weeks of treatment. Data for LS mean rate of change in BMI over 104 weeks are shown in Table S5 in the supplement. A Kaplan-Meier plot of time to first PEx is shown in Figure S3 in the supplement.

\section{DISCUSSION}

Ivacaftor was well tolerated with no new safety concerns observed in pwCF aged 6 years and older with non-G551D-CFTR gating mutations, the $R 117 H$-CFTR mutation, or other residual function mutations. Safety results were consistent with ivacaftor's well-established safety profile demonstrated in clinical studies and by real-world evidence [2-11].

Ivacaftor showed durable clinical benefit across the three parent-study subgroups, improving lung function, sweat chloride concentration, and CFQ-R RD scores for up to 2 years of treatment. On average, BMI showed a 
Table 2 Safety summary of adverse events

\begin{tabular}{ll}
\hline$n$ (\%) & Overall $(\boldsymbol{N}=\mathbf{1 2 1})$ \\
\hline Participants with any AEs & $117(96.7)$ \\
AEs related to the study medication $^{a}$ & $24(19.8)$ \\
AEs leading to death & 0 \\
Participants with any SAEs & $27(22.3)$ \\
SAEs related to the study medication ${ }^{\mathrm{b}}$ & $2(1.7)$ \\
AEs leading to study drug interruption & $19(15.7)$ \\
AEs leading to study drug withdrawal & $3(2.5)$ \\
AEs occurring in $\geq 10 \%$ of participants & \\
Infective PEx of CF & $56(46.3)$ \\
Cough & $41(33.9)$ \\
Headache & $23(19.0)$ \\
Sinus congestion & $22(18.2)$ \\
Sputum increased & $22(18.2)$ \\
Nasopharyngitis & $21(17.4)$ \\
Sinusitis & $19(15.7)$ \\
Oropharyngeal pain & $18(14.9)$ \\
Viral upper respiratory tract infection & $17(14.0)$ \\
Nasal congestion & $17(14.0)$ \\
Constipation & $17(14.0)$ \\
Diarrhea & $17(14.0)$ \\
Pyrexia & $17(14.0)$ \\
Upper respiratory tract infection & $15(12.4)$ \\
Abdominal pain & $13(10.7)$ \\
\hline
\end{tabular}

$A E$ adverse event, $C F$ cystic fibrosis, $P E x$ pulmonary exacerbation, $S A E$ serious adverse event

a Includes AEs deemed "related" or "possibly related" to the study medication

b One possibly related SAE of infective PEx of CF, and one possibly related SAE of sinusitis

robust increase over 2 years in participants 18 years and older in parent-study subgroup 110. For other parent-study subgroups, the sample sizes were too low to interpret the numerically positive increases. Similarly, the sample sizes were too low to interpret BMI-forage z-score (Table S5 in the supplement).
As CF is a chronic progressive disease, lung function is expected to decline (by approximately $1-3 \%$ per year) $[15,16]$ due to increasing age, number, and severity of PEx, nutritional status, presence of lung infections, and other factors. Despite this underlying progression, the magnitude of the increase in lung function in this long-term (up to 104 weeks) extension 
Study 110

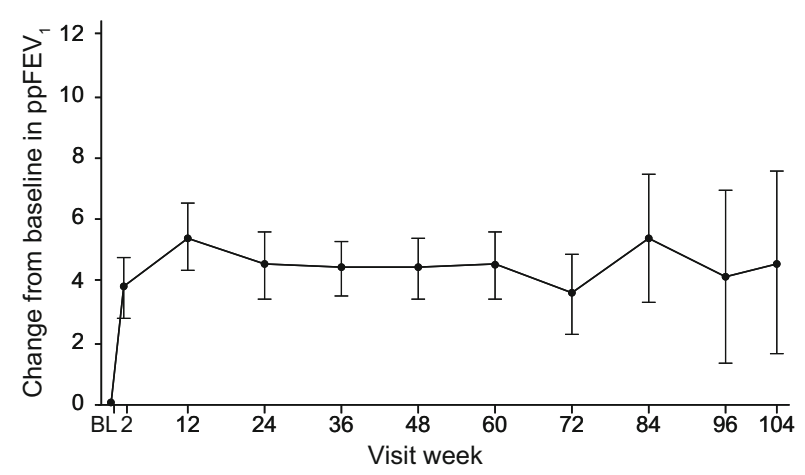

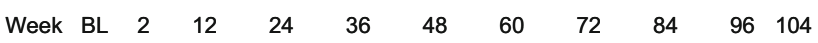
$\begin{array}{llllllllllll}n & 65 & 65 & 63 & 62 & 60 & 58 & 58 & 50 & 40 & 28 & 20\end{array}$

\section{Study 111}
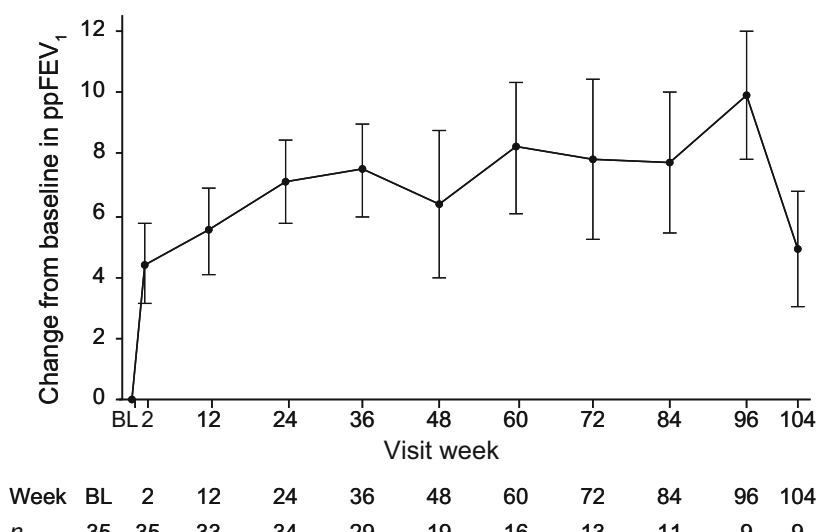

$\begin{array}{llllllllllll}n & 35 & 35 & 33 & 34 & 29 & 19 & 16 & 13 & 11 & 9 & 9\end{array}$

Study 113

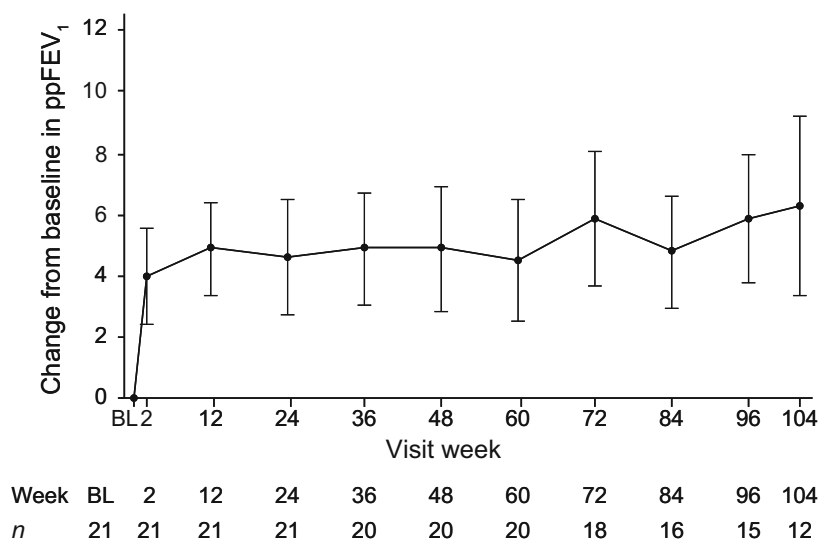

Fig. 2 Absolute change from baseline in $\mathrm{ppFEV}_{1}$ in Extension Study 112 by parent-study subgroup. Data are least-squares means based on a mixed-effects model for repeated measures, and error bars indicate standard errors. $B L$ baseline, $p p F E V_{1}$ percent predicted forced expiratory volume in $1 \mathrm{~s}$ 
Study 110

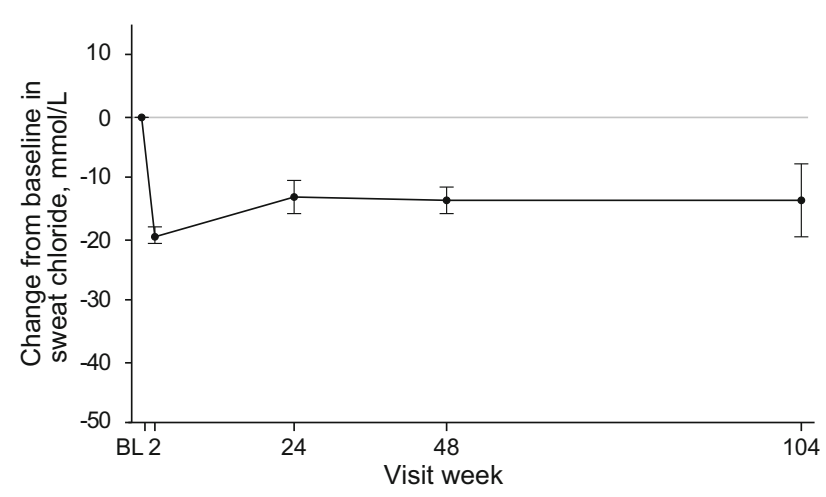

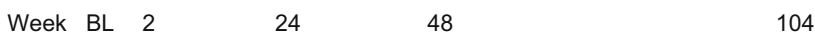

$\begin{array}{llllll}n & 59 & 59 & 56 & 50 & 15\end{array}$

Study 111

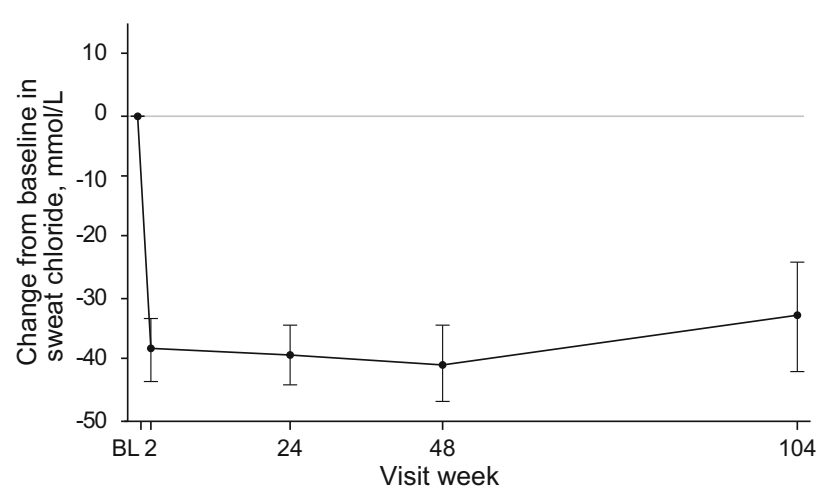

$\begin{array}{llllll}\text { Week } & \text { BL } & 2 & 24 & 48 & 104\end{array}$

$\begin{array}{llllll}n & 33 & 31 & 33 & 19 & 9\end{array}$

Study 113

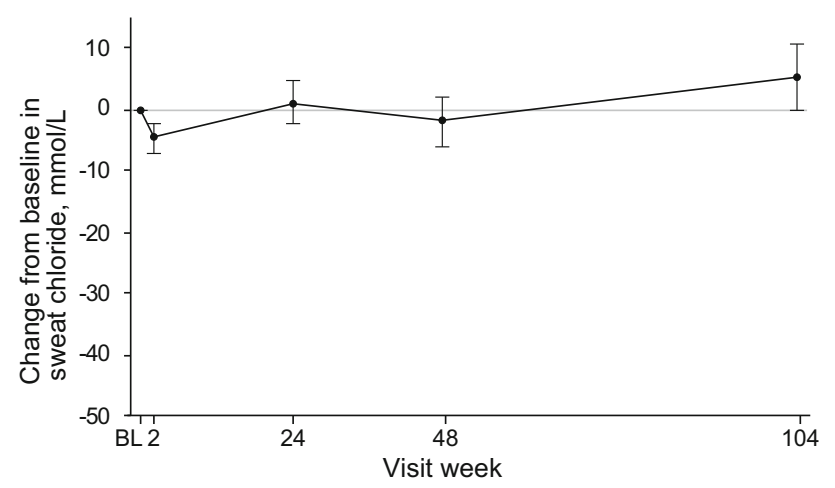

$\begin{array}{lllllc}\text { Week } & \mathrm{BL} & 2 & 24 & 48 & 104 \\ n & 21 & 21 & 20 & 19 & 11\end{array}$

Fig. 3 Absolute change from baseline in sweat chloride concentration in Extension Study 112 by parent-study subgroup. Data are least-squares means based on a mixed- effects model for repeated measures, and error bars indicate standard errors. Solid gray line (zero) represents no change from Extension Study 112 baseline. BL baseline 
study is consistent with the expected outcomes based on data from the parent studies $(110,111$, and 113). Moreover, outcomes from this extension study are consistent with outcomes from other long-term studies demonstrating that participants treated with ivacaftor have better-preserved lung function [4, 17].

The genotypes of participants in the parentstudy subgroups are associated with different levels of CF severity, as seen by the higher baseline mean sweat chloride concentrations in participants from Study 111 and by the lower mean concentrations in Study 110 and Study 113; in Study 113, the mean sweat chloride concentration was below the $60 \mathrm{mmol} / \mathrm{l} \mathrm{diag-}$ nostic threshold (Table 1) [18]. These differences at baseline may have contributed to variations in response, which is consistent with the outcomes in the parent studies. For example, the magnitude of response in sweat chloride concentration was greatest in Study 111, in which participants had the highest baseline mean concentration.

This open-label extension study has several potential limitations. Using a voluntary rollover cohort risks introducing a selection bias. However, the extremely high participation rate (121/ 133 [91\%]) suggests that any selection bias was limited. A further limitation is the lack of a comparator group, which can make the interpretation of outcomes more difficult. Moreover, almost half of the participants (59/121 [49\%]) withdrew from the study because ivacaftor became commercially available to them. Despite this attrition, which increased variability and the impact of events such as PEx, the outcomes in this extension study are generally consistent with the results from the parent studies.

\section{CONCLUSIONS}

Ivacaftor was generally safe and well tolerated for up to 104 weeks of treatment in pwCF with ivacaftor-responsive mutations other than G551D. No new safety concerns were identified. The pattern of improvement across efficacy endpoints was durable and generally consistent with outcomes from the parent studies.
Collectively, these data suggest that ivacaftor is likely to significantly slow progression of CF lung disease and improve other long-term outcomes, including quality of life.

\section{ACKNOWLEDGEMENTS}

The authors thank the people with cystic fibrosis and their families, the trial coordinators, and the investigators for their contributions to the trials.

Funding. This study and its publication (Rapid Service Fee) were supported by Vertex Pharmaceuticals Incorporated.

Medical Writing and Editorial Assistance. Editorial coordination and support were provided by Paulina Phan, who is completing a fellowship program funded by Vertex Pharmaceuticals Incorporated and in collaboration with Northeastern University. Medical writing and editorial support were provided under the direction of the authors by Amos Race, PhD, of ArticulateScience LLC, with support from Vertex Pharmaceuticals Incorporated.

Authorship. All named authors meet the International Committee of Medical Journal Editors (ICMJE) criteria for authorship for this article, take responsibility for the integrity of the work as a whole, and have given their approval for this version to be published.

Disclosures. All authors received nonfinancial support (assistance with manuscript preparation) from ArticulateScience LLC, which received funding from Vertex Pharmaceuticals Incorporated. Additional disclosures are as follows: ST, CD, and MH: employees of Vertex Pharmaceuticals Incorporated and may own stock or stock options in Vertex Pharmaceuticals Incorporated. JMP: grants from Vertex Pharmaceuticals Incorporated. KDeB: grants from Galapagos and Vertex Pharmaceuticals Incorporated; personal fees from Boehringer, Protalix, Raptor, Teva, NovaBiotics, Eloxx, Vertex Pharmaceuticals Incorporated, Galapagos, 
and Chiesi. JAN: personal fees from Vertex Pharmaceuticals Incorporated. RBM: personal fees from Proteostasis, Aridis, Mayne, Zambon, PULMATRiX, and Regeneron.

Compliance with Ethics Guidelines. This study was conducted in accordance with good clinical practices, as described in the International Council for Harmonisation guidelines, and is consistent with the World Medical Association Declaration of Helsinki and applicable regional laws and regulations. The study protocol, informed consent form, and other necessary documents were reviewed and approved by an independent ethics committee or institutional review board for each study site before initiation of the study at that site (Table S1 in the supplement). Written informed consent was obtained from or for (with assent, where applicable) each participant on (or before) the day 1 visit.

Data Availability. Vertex is committed to advancing medical science and improving patient health. This includes the responsible sharing of clinical trial data with qualified researchers. Proposals for the use of these data will be reviewed by a scientific board. Approvals are at the discretion of Vertex and will be dependent on the nature of the request, the merit of the research proposed, and the intended use of the data. Please contact CTDS@vrtx.com if you would like to submit a proposal or need more information.

Open Access. This article is licensed under a Creative Commons Attribution-NonCommercial 4.0 International License, which permits any non-commercial use, sharing, adaptation, distribution and reproduction in any medium or format, as long as you give appropriate credit to the original author(s) and the source, provide a link to the Creative Commons licence, and indicate if changes were made. The images or other third party material in this article are included in the article's Creative Commons licence, unless indicated otherwise in a credit line to the material. If material is not included in the article's Creative Commons licence and your intended use is not permitted by statutory regulation or exceeds the permitted use, you will need to obtain permission directly from the copyright holder. To view a copy of this licence, visit http://creativecommons.org/licenses/by$\mathrm{nc} / 4.0 /$.

\section{REFERENCES}

1. Ramsey BW, Davies J, McElvaney NG, et al. A CFTR potentiator in patients with cystic fibrosis and the G551D mutation. N Engl J Med. 2011;365:1663-722. https://doi.org/10.1056/NEJMoa1105185.

2. Kalydeco ${ }^{\circledR}$ (ivacaftor) [prescribing information]. Boston, MA: Vertex Pharmaceuticals Incorporated; 2019.

3. Davies JC, Cunningham S, Harris WT, et al. Safety, pharmacokinetics, and pharmacodynamics of ivacaftor in patients aged 2-5 years with cystic fibrosis and a CFTR gating mutation (KIWI): an open-label, single-arm study. Lancet Respir Med. 2016;4:107-15. https://doi.org/10.1016/S2213-2600(15)00545-7.

4. Volkova N, Moy K, Evans J, et al. Disease progression in patients with cystic fibrosis treated with ivacaftor: data from national US and UK registries. J Cyst Fibros. 2020;19(1):68-79. https://doi.org/10. 1016/j.jcf.2019.05.015.

5. Davies JC, Wainwright CE, Canny GJ, et al. Efficacy and safety of ivacaftor in patients aged 6 to 11 years with cystic fibrosis with a G551D mutation. Am J Respir Crit Care Med. 2013;187:1219-25. https:// doi.org/10.1164/rccm.201301-0153OC.

6. De Boeck K, Munck A, Walker S, et al. Efficacy and safety of ivacaftor in patients with cystic fibrosis and a non-G551D gating mutation. J Cyst Fibros. 2014;13:674-80. https://doi.org/10.1016/j.jcf.2014. 09.005 .

7. McNamara JJ, McColley SA, Marigowda G, et al. Safety, pharmacokinetics, and pharmacodynamics of lumacaftor and ivacaftor combination therapy in children aged 2-5 years with cystic fibrosis homozygous for F508del-CFTR: an open-label phase 3 study. Lancet Respir Med. 2019;7:325-35. https:// doi.org/10.1016/S2213-2600(18)30460-0.

8. Moss RB, Flume PA, Elborn JS, et al. Efficacy and safety of ivacaftor in patients with cystic fibrosis who have an Arg117His-CFTR mutation: a doubleblind, randomised controlled trial. Lancet Respir Med. 2015;3:524-33. https://doi.org/10.1016/ S2213-2600(15)00201-5. 
9. Rosenfeld M, Wainwright CE, Higgins $\mathrm{M}$, et al. Ivacaftor treatment of cystic fibrosis in children aged 12 to less than 24 months and with a CFTR gating mutation (ARRIVAL): a phase 3 single-arm study. Lancet Respir Med. 2018;6:545-53. https:// doi.org/10.1016/S2213-2600(18)30202-9.

10. Bessonova L, Volkova N, Higgins M, et al. Data from the US and UK cystic fibrosis registries support disease modification by CFTR modulation with ivacaftor. Thorax. 2018;73:731-40. https://doi.org/10. 1136/thoraxjnl-2017-210394.

11. Hubert D, Dehillotte C, Munck A, et al. Retrospective observational study of French patients with cystic fibrosis and a Gly551Asp-CFTR mutation after 1 and 2 years of treatment with ivacaftor in a real-world setting. J Cystic Fibros. 2018;17:89-95. https://doi.org/10.1016/j.jcf.2017.07.001.

12. Kalydeco ${ }^{\circledR}$ (ivacaftor) [summary of product characteristics]. Dublin, Ireland: Vertex Pharmaceuticals (Ireland) Limited; 2019.

13. Guimbellot J, Solomon GM, Baines A, et al. Effectiveness of ivacaftor in cystic fibrosis patients with non-G551D gating mutations. J Cyst Fibros. 2019;18(1):102-9. https://doi.org/10.1016/j.jcf. 2018.04.004.
14. Nick JA, St Clair C, Jones MC, Lan L, Higgins M, Team VXS. Ivacaftor in cystic fibrosis with residual function: Lung function results from an N-of-1 study. J Cyst Fibros. 2020;19:91-8. https://doi.org/ 10.1016/j.jcf.2019.09.013.

15. Konstan MW, Wagener JS, Vandevanter DR, et al. Risk factors for rate of decline in FEV1 in adults with cystic fibrosis. J Cyst Fibros. 2012;11:405-11. https://doi.org/10.1016/j.jcf.2012.03.009.

16. Konstan MW, Morgan WJ, Butler SM, et al. Risk factors for rate of decline in forced expiratory volume in one second in children and adolescents with cystic fibrosis. J Pediatr. 2007;151(134-9):e1. https://doi.org/10.1016/j.jpeds.2007.03.006.

17. McKone EF, Borowitz D, Drevinek P, et al. Longterm safety and efficacy of ivacaftor in patients with cystic fibrosis who have the Gly551Asp-CFTR mutation: a phase 3, open-label extension study (PERSIST). Lancet Respir Med. 2014;2:902-10. https://doi.org/10.1016/S2213-2600(14)70218-8.

18. Farrell PM, White TB, Ren CL, et al. Diagnosis of cystic fibrosis: consensus guidelines from the Cystic Fibrosis Foundation. J Pediatr. 2017;181S:S4-S15. e1. https://doi.org/10.1016/j.jpeds.2016.09.064. 